\title{
Philosophiques
}

Strauss, Leo et Cropsey, Joseph (dir.), Histoire de la philosophie politique, traduit de l'anglais par O. Sedeyn, Paris, PUF (coll. « Léviathan "), 1994, $1076 \mathrm{p}$.

Strauss, Leo, Qu'est-ce que la philosophie politique ?, traduit de l'anglais par O. Sedeyn, Paris, PUF (coll. « Léviathan »), 1992.

\section{Pierre Gravel}

Volume 24, numéro 2, automne 1997

URI : https://id.erudit.org/iderudit/027462ar

DOI : https://doi.org/10.7202/027462ar

Aller au sommaire du numéro

Éditeur(s)

Société de philosophie du Québec

\section{ISSN}

0316-2923 (imprimé)

1492-1391 (numérique)

Découvrir la revue

\section{Citer ce compte rendu}

Gravel, P. (1997). Compte rendu de [Strauss, Leo et Cropsey, Joseph (dir.), Histoire de la philosophie politique, traduit de l'anglais par O. Sedeyn, Paris, PUF (coll. « Léviathan »), 1994, 1076 p. / Strauss, Leo, Qu'est-ce que la philosophie politique ?, traduit de l'anglais par O. Sedeyn, Paris, PUF (coll. " Léviathan »), 1992.] Philosophiques, 24(2), 435-436. https://doi.org/10.7202/027462ar d'utilisation que vous pouvez consulter en ligne. 
Strauss, Leo et Cropsey, Joseph (dir.), Histoire de la philosophie politique, traduit de l'anglais par O. Sedeyn, Paris, PUF (coll. "Léviathan "), 1994, $1076 \mathrm{p}$.

Strauss, Leo, Qu'est-ce que la philosophie politique?, traduit de l'anglais par O. Sedeyn, Paris, PUF (coll. « Léviathan »), 1992.

L'œuvre de Leo Strauss commence d'être connue en français. Auteur de quinze ouvrages, dont trois publiés en allemand et douze en anglais, Leo Strauss (1899-1973) a quitté l'Allemagne en 1932 pour des raisons que l'on devine fort bien et, cela après avoir suivi des cours, entre autres, de Husserl et de Heidegger, après aussi avoir travaillé comme chercheur dans un institut d'études juives de Berlin, il finit par s'installer à Chicago principalement, où il enseigna de 1938 jusqu'à sa mort, en 1973. Ce qu'il convient d'abord de souligner de ces deux ouvrages dont le premier, l' Histoire, connut trois éditions différentes et augmentées de 1963 à 1987, alors que le second fut publié pour la première fois en 1957, c'est que ces deux ouvrages, le premier surtout, témoignent éminemment de la valeur, qui commence d'être affirmée, que Leo Strauss attribuait dans son métier à l'enseignement conçu à la manière allemande comme formation (Bildung). Leo Strauss a passé la majeure partie de sa carrière à former des générations d'étudiants dans le domaine de la philosophie politique conçue de manière historique, non pas historiale au sens de Heidegger, c'est-à-dire au sens où une même question, celle du sens de l'être, se voit identiquement posée chez différents auteurs, mais au sens où chaque auteur étudié se voit considéré de l'intérieur au sein de l'époque qui est la sienne, époque au sens de l'aire théorique plus que de l'“ époque • husserlienne. Je tiens à souligner cette valeur attribuée à la formation pédagogique au sens noble du terme, en cette époque, la nôtre, où les enseignants universitaires se voient beaucoup plus évalués en fonction de la stricte quantité de leurs publications dans des revues dites scientifiques, que par leur enseignement. Une université digne de ce nom pour les générations à venir ne devrait-elle pas d'abord former, et prendre en considération la question même de la formation, ce qui s'y fait de toute façon?

Les deux livres ici recensés, et traduits presque coup sur coup par Olivier Sedeyn, sont composés de manière analogue : de courtes monographies rassemblées autour d'une question qui se voit approfondie, littéralement creusée, chez des auteurs différents, chacun, comme nous l'avons dit, envisagé dans ses conditions et ses circonstances historiques internes. Et ce qui se manifeste le plus globalement en ce genre de travail, trait qui est d'ailleurs souligné par le traducteur, c'est que nous sommes peut-être, dans ce domaine de l'existence qui s'appelle le politique, plus près paradoxalement des Anciens que des Modernes dans l'abord même des questions. Et l'un des traits par où cette dernière perspective se manifeste au mieux, c'est que Leo 
Strauss lui-même se voyait de plus en plus avancer dans cette traversée de l'histoire de la philosophie politique qui a été la sienne par un renouvellement de la question du politique dans une perspective aristotélicienne. Ce trait est remarquablement mis en relief dans le dernier article de l'Histoire qui est consacré à ce que nous appellerions le a bougé a de son œuvre : une étude sur les variations dues à l'approfondissement des questions traitées.

L'Histoire, sur laquelle nous insistons à cause de son caractère moins immédiatement spécialisé, mais, ne nous y trompons pas, tout aussi hautement et fortement philosophique, se présente comme un fort volume de 1076 pages, contenant trente-huit études sous forme de monographies d'autant d'auteurs différents considérés dans leur rapport au politique, plus précisément dans le rapport interne qui se dégage en fonction de la question du politique. C'est ainsi, par exemple, que s'il peut paraître évident de trouver dans un ouvrage collectif de ce genre des études consacrées à Platon, texte écrit par Leo Strauss, ou à Machiavel, lui aussi écrit par Strauss, ou encore à Rousseau, rédigé par Allan Bloom, un ancien étudiant de Strauss récemment décédé, quel n'est pas notre étonnement de trouver des études consacrées à des auteurs non habituellement considérés comme strictement politiques, tels que Augustin et Thomas d'Aquin, Martin Luther et Jean Calvin, mais aussi Bacon, Descartes et, à la toute fin, Husserl et Heidegger ! Cet ouvrage, dont nous tenons à souligner la richesse, se termine sur un épilogue consacré à la pensée politique de Leo Strauss, texte écrit quelque temps après sa mort, et inséré à titre d'hommage posthume. Soulignons enfin, dans cette Histoire, le très riche index qui en fait un véritable instrument de travail.

Le Qu'est-ce que la philosophie politique ? rassemble quinze chapitres regroupant des articles publiés entre 1945 et 1958, suivis de seize a jugements critiques a, en fait des comptes rendus publiés pendant la même époque. Textes intéressants dans la mesure où l'on voit, en quelque sorte, Leo Strauss affronter ses contemporains. Mais le plus important est de remarquer que tous ces textes ont été écrits et publiés pendant la période la plus mûre et la plus féconde de la vie de notre auteur, alors âgé entre quarante et soixante ans, et que tous ces textes s'attaquent à la question du fondement même de la philosophie politique envisagée selon l'angle historique, et non pas historiciste, de son auteur. Ce sont donc des textes théoriques pour la plupart, même si certains abordent des problèmes apparemment techniques mais significativement importants, comme ceux soulevés par des textes de Xénophon, de Fârâbi, de Maïmonide, de Hobbes et de Locke. L'érudition, et dans ce cas-ci l'extrême érudition, peut donc côtoyer et épauler le travail de la pensée dans sa sobriété la plus grande. La force de Leo Strauss tient à cette gageure dont il nous a démontré la fécondité : s'en tenir chaque fois, et dans chaque cas, à ce que peut révéler, et permettre de penser, une lecture interne de l'auteur considéré. Et à nouveau, il convient de souligner que cet ouvrage est lui aussi suivi d'un remarquable index qui en facilite de beaucoup la lecture et le travail.

Pierre Gravel

Département de philosophie

Université de Montréal 